\title{
Residual-free bubble shape functions used in BEM for the stability of the solution of the convective-diffusion transport equation
}

\author{
P. Kocutar, L. Škerget \& J. Ravnik \\ Faculty of Mechanical Engineering, University of Maribor, Slovenia
}

\begin{abstract}
In this paper we focus on oscillation and instability of the solution of the convective-diffusive equation depending on the Peclet number. For that purpose, different types of mesh elements and shape functions have been used. For the stabilization of the solution of the convective-diffusive equation for high Peclet numbers, we employed high-order polynomial shape functions, namely residualfree bubble functions. The numerical scheme is based on BEM. For solving the linear homogeneous part of the partial differential equation, the Laplace fundamental solution has been used.

We compared quadratic nine-node domain element using Lagrangian shape functions, linear four-node domain element using Lagrangian shape functions and linear four-node domain element using fourth-order bubble enriched functions. Numerical results obtained with linear Lagrangian shape function and bubble enriched functions are compared with the analytical solution.

Residual-free bubble functions add stability to simulation and despite the fact that less nodes are used in the domain element, the results are comparable and in some cases even better than the quadratic nine-node domain element. The boundary element method with usage of bubble-enriched functions can resolve problems of convective-diffusion and obtain stable and accurate solutions for this type of governing equations, which are being represented in several types of transport phenomena.
\end{abstract}

Keywords: boundary element method, residual free bubble, upwinding techniques, convective-diffusion equation. 


\section{Introduction}

It is known that the solution of the convective-diffusive equation for high Peclet numbers is unstable for standard numerical schemes. Samec and Škerget [1] focused on numerical solution of a diffusive-convective transport equation for reacting flows based on boundary-domain integral formulation for diffusionconvective fundamental solution. Nassehi and Parvazinia [2] studied instabilities of convective-diffusion equations using a variational multiscale finite element scheme. They used discretization based on the utilization of bubble function enriched finite elements. Residual free bubble method for stability of convectiondiffusion problems was further used by Franca et al. [3]. They used the Galerkin finite element method for partial differential equations in two dimensions and the finite-dimensional space consists of piecewise polynomials enriched with bubble functions. Furthermore, Franca and Nesliturk [4] employed two-level finite element method with combination of enriched residual-free bubble functions for simulation of Navier-Stokes equations for incompressible fluid.

\section{Governing equations}

\subsection{Diffusion-convective equation}

Steady state nonlinear diffusion-convective equation [5], which describes time independent transport, can be written as

$$
P e_{j} \frac{\partial T}{\partial x_{j}}=\frac{\partial^{2} T}{\partial x_{j} \partial x_{j}} \quad \text { in } \Omega
$$

where $P e_{j}$ is the Peclet number defined with velocity component $v_{j}$, characteristic length scale $h$ and diffusivity $\alpha$ as $P e_{j}=\frac{v_{j} l}{\alpha}$. The variable $T(\vec{r})$ can be used as a temperature in heat transfer problems, concentration in dispersion processes or vorticity in fluid dynamics.

Equation (1) can be treated as a nonhomogeneous linear elliptic Poisson's type of partial differential equation (PDE), therefore we may write

$$
\mathcal{L}[T]+b=\frac{\partial^{2} T}{\partial x_{j} \partial x_{j}}+b=0 \quad \text { in } \Omega .
$$

In equation (2) $\mathcal{L}[\cdot]$ represent linear elliptic Laplace differential operator and $b$ represents pseudo body force for convective term.

We can formulate corresponding boundary-domain integral by applying a weighted residual technique by Green's theorems for scalar functions. The Green's function is known function of a geometry capturing only the diffusion of the transport process. Thus, we rewrite equation (2)

$$
c(\xi) T(\xi)+\int_{\Gamma} T q^{*} d \Gamma=\int_{\Gamma} q T^{*} d \Gamma-\int_{\Omega} P e_{j} \frac{\partial T}{\partial x_{j}} T^{*} d \Omega,
$$


where $\xi$ is the source point, $T^{*}$ is the fundamental solution of the Laplace operator and $q^{*}$ its gradient. $q$ is the function flux. In convective term, the domain integral involves the partial derivatives of the field function $T$. In order to eliminate the derivative, we use the Gaussian divergence theorem on the convective domain integral. Thus the divergence operator is shifted to the fundamental solution, we may write an integral formulation without derivatives of fields function in domain integral as:

$$
c(\xi) T(\xi)+\int_{\Gamma} T q^{*} d \Gamma=\int_{\Gamma} q T^{*} d \Gamma-\int_{\Gamma} T P e_{n} T^{*} d \Gamma+\int_{\Omega} T P e_{j} q_{j}^{*} d \Omega
$$

In equation (4) $q_{j}^{*}$ is defined as $q_{j}^{*}=\frac{\partial T^{*}}{\partial x_{j}}$ and $P e_{n}$ is Peclet number calculated with normal velocity component to the boundary and defined as $v_{n}=\vec{v} \cdot \vec{n}$.

\subsubsection{Discretization}

We write the corresponding boundary-domain integral in a discretized form for the numerical solution of diffusion-convective equation. Integrals over the boundary and domain are approximated by a summation of integrals over individual boundary elements and internal cells respectively. Thus we may write discretization for linear integral statement (4) as

$$
\begin{aligned}
c(\xi) T(\xi)+\sum_{e=1}^{E} \int_{\Gamma_{e}} T q^{*} d \Gamma=\sum_{e=1}^{E} \int_{\Gamma_{e}} q T^{*} d \Gamma & -\sum_{e=1}^{E} \int_{\Gamma_{e}} T P e_{n} T^{*} d \Gamma \\
& +\sum_{c=1}^{C} \int_{\Omega_{c}} T P e_{j} q_{j}^{*} d \Omega,
\end{aligned}
$$

where $E$ is number of boundary elements and $C$ is a number of internal cells.

The product of field function for each boundary element or internal cell may be approximated with shape functions. We employed boundary shape functions $\Phi_{B i}$ and $\Phi_{i}$ and domain shape functions $\phi_{B i}$ yielding

$$
\begin{array}{r}
c(\xi) T(\xi)+\sum_{e=1}^{E} \int_{\Gamma_{e}} \sum_{i} \Phi_{B i} T_{i} q^{*} d \Gamma=\sum_{e=1}^{E} \int_{\Gamma_{e}} \sum_{i} \Phi_{i} q_{i} T^{*} d \Gamma \\
-\sum_{e=1}^{E} \int_{\Gamma_{e}} \sum_{i} \Phi_{B i}\left(T P e_{n}\right)_{i} T^{*} d \Gamma+\sum_{c=1}^{C} \int_{\Omega_{c}} \sum_{i} \phi_{B i}\left(T P e_{j}\right)_{i} q_{j}^{*} d \Omega .
\end{array}
$$




\section{Numerical method}

\subsection{Setting up the system of equations}

For numerical method, we used macro element BEM [6]. We solve equation (6) for each mesh element. In each quadratic element with quadratic interpolation for function and linear for fluxes, there are 9 nodes for function and 8 nodes for fluxes, see Figure 1 left. For setting up the system of equation, the source point is set in all of those nodes $(9+8=17)$. For that type of cell (quadratic interpolation for function and linear for fluxes) we have together 17 equation for each domain cell. Since we want to reduce size of matrixes, we introduce four-sided element with linear interpolation for functions and constant for fluxes. That type of cells has 4 nodes for functions and 4 nodes for fluxes, see Figure 1 right. Thus, we get instead of 17 only 8 equation for each cell. For compensation of disadvantages of linear 4 node cell, for functions, in comparison with quadratic 9 node cell, we introduce upwinding method, which from computational aspect of view, obtain same number of equations as linear interpolation and require same memory space.

Because neighbouring cells share nodes and boundary conditions on the other boundaries of the domain are prescribed, we get an overdetermined system of equations. For that reason we used in our numerical algorithm least squares manner solver.
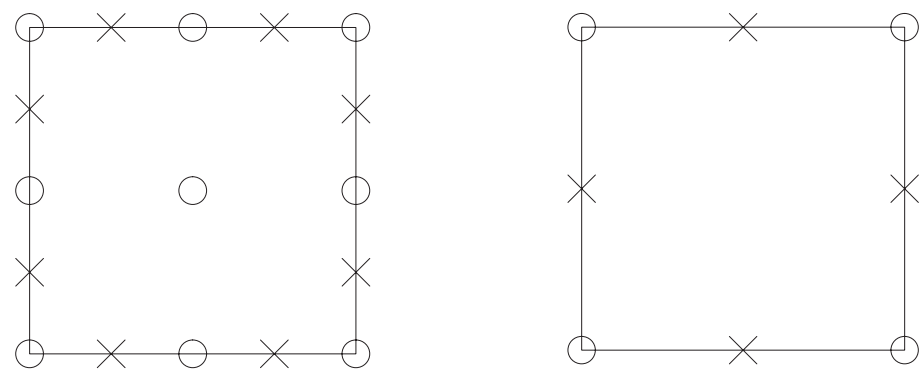

Figure 1: Left: four-sided cell with quadratic interpolation for functions and linear for fluxes. Right: four-sided cell with linear interpolation for functions and constant fluxes.

\subsection{Shape functions}

We obtain two-dimensional domain $\Omega$ with one-dimensional boundary $\Gamma$. Domain is split into domain cells and boundary into boundary elements [7,8].

\subsection{Upwinding techniques}

For the purpose of stabilization of boundary element solution we decide to employ one of few upwinding methods [9]. Over time, various methods, which 
can stabilize numerical solution have been developed: streamline upwind PetrowGalerkin method (SUPG), direct static condensation method (STC), Galerkin least squares method (GLS) and residual free bubble method (RFB).

\subsubsection{Residual free bubble (RFB)}

Bubble functions are typically high-order polynomials, which are effective within an element or cell, but their effect vanishes at the element boundaries. RFB functions are derived via the analytical solution of these equations in conjunction with boundary conditions, and should be strongly satisfy them within each element. Thus, the derivation of the bubble functions for a given problem is based on the analytical solution of its governing differential equation posed within each element.

RFB shape functions are linear, i.e. they require two node per mesh element. Their shape is determined by the Peclet number. For zero Peclet number $P e=0$, they revert back to linear Lagrangian shape functions. For non-zero Peclet number $P e \neq 0$ their shape is governed by the analytical solution of convective-diffusion equation.

The RFB shape functions can be used to interpolate function value within a mesh element using

$$
T(\xi)=T_{1} \cdot \Phi_{B 1}(\xi)+T_{2} \cdot \Phi_{B 2}(\xi),
$$

where $\xi$ is the local coordinate system.

\subsubsection{Derivation of shape functions}

We can write two-dimensional convective-diffusion equation (1) in a nondimensional form in planar $(x, y)$ coordinate system as

$$
P e_{x} \frac{\partial T}{\partial x}+P e_{y} \frac{\partial T}{\partial y}-\frac{\partial^{2} T}{\partial x^{2}}-\frac{\partial^{2} T}{\partial y^{2}}=0 .
$$

Values of equation (8) at nodal points provide analytically obtained shape functions $\Phi_{B 1}$ and $\Phi_{B 2}$. If we substitute $T$ with $\Phi_{B 1}$ and $\Phi_{B 2}$, we get

$$
\begin{aligned}
& -\frac{d^{2} \Phi_{B 1}}{d x^{2}}+P e_{j} \frac{d \Phi_{B 1}}{d x}=0 \text { for } x \in[0-l], \\
& \Phi_{B 1}(0)=1, \quad \Phi_{B 1}(l)=0
\end{aligned}
$$

and

$$
\begin{aligned}
& -\frac{d^{2} \Phi_{B 2}}{d x^{2}}+P e \frac{d \Phi_{B 2}}{d x}=0 \text { for } x \in[0-l] \\
& \Phi_{B 2}(0)=0, \quad \Phi_{B 2}(l)=1,
\end{aligned}
$$


where $l$ is the element length. Using local coordinate system, solution of above equations can be derived into bubble shape functions

$$
\Phi_{B 1}=\frac{e^{x P e}-e^{l P e}}{1-e^{l P e}}, \quad \Phi_{B 2}=\frac{1-e^{x P e}}{1-e^{l P e}} .
$$

We expand the exponential function $e^{x}$ in to a Taylor series as:

$$
e^{x}=1+x+\frac{x^{2}}{2 !}+\frac{x^{3}}{3 !}+\ldots
$$

Bubble functions are sum of Lagrangian shape functions $\Phi_{L}$ and enriched appendix $\Phi_{E}, \Phi_{B}=\Phi_{L}+\Phi_{E}$. We keep only the first three terms in Taylor series expansion. Thus we can, with introduction of local coordinate system $\xi(-1,+1)$ and defining $x=\frac{l}{2}(1+\xi)$, write bubble shape function of second-order as

$$
\Phi_{B 1}=\frac{1}{2}(1-\xi)-\left(b\left(1-\xi^{2}\right)\right), \quad \Phi_{B 2}=\frac{1}{2}(1+\xi)+\left(b\left(1-\xi^{2}\right)\right),
$$

where $b$ is defined as

$$
b=\frac{l P e}{8(1+0.5 l P e)} .
$$

Alternatively, keeping first five terms instead of three in Taylor series expansion we get fourth-order bubble shape functions

$$
\begin{aligned}
& \Phi_{B 1}=\frac{1}{2}(1-\xi)-\left(b_{1}\left(1-\xi^{2}\right)+b_{2}(1+\xi)\left(1-\xi^{2}\right)+b_{3}\left(1-\xi^{2}\right)^{2}\right), \\
& \Phi_{B 2}=\frac{1}{2}(1+\xi)+\left(b_{1}\left(1-\xi^{2}\right)+b_{2}(1+\xi)\left(1-\xi^{2}\right)+b_{3}\left(1-\xi^{2}\right)^{2}\right),
\end{aligned}
$$

where parameters $b_{1}, b_{2}$ and $b_{3}$ are defined as

$$
\begin{gathered}
b_{1}=\frac{\frac{1}{2 !} P e^{2} l^{2}+\frac{1}{3 !} P e^{3} l^{3}+\frac{1}{4 !} P e^{4} l^{4}}{4\left(P e l+\frac{P e^{2} l^{2}}{2 !}+\frac{P e^{3} l^{3}}{3 !}+\frac{P e^{4} l^{4}}{4 !}\right)}, \\
b_{2}=\frac{\frac{1}{3 !} P e^{3} l^{3}+\frac{1}{4 !} P e^{4} l^{4}}{8\left(P e l+\frac{P e^{2} l^{2}}{2 !}+\frac{P e^{3} l^{3}}{3 !}+\frac{P e^{4} l^{4}}{4 !}\right)}, \\
b_{3}=\frac{\frac{1}{4 !} P e^{4} l^{4}}{16\left(P e l+\frac{P e^{2} l^{2}}{2 !}+\frac{P e^{3} l^{3}}{3 !}+\frac{P e^{4} l^{4}}{4 !}\right)} .
\end{gathered}
$$

Analogously to the above derivation of RFB shape functions for boundary element $\Phi_{B i}$, we derive RFB shape functions for domain cell $\phi_{B i}$.

In described two-node linear element, the sign of bubble coefficient is different for the beginning and end nodes. The opposite signs of the bubble functions affect the convection term in the convective-diffusion equation and consequently on the scope of bubble function. Figure 2 shows 2 node boundary element, enriched with residual free bubble function of 4 th order. 


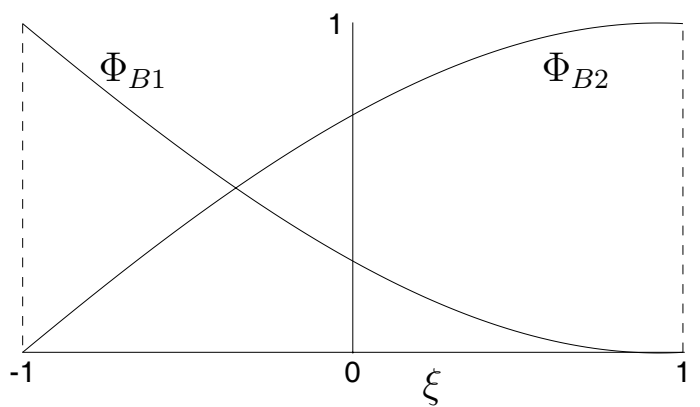

Figure 2: Bubble enriched shape function.

\section{Numerical tests}

\subsection{Entry flow problem}

We tested our numerical scheme on entry flow problem. For entry flow problem we defined following boundary conditions: uniform velocity on whole domain, temperature $T=1$ on the inlet, $T=0$ on the outflow and adiabatic boundary conditions $d T / d y=0$ on the upper and lower plate. Boundary conditions are shown in Figure 3.

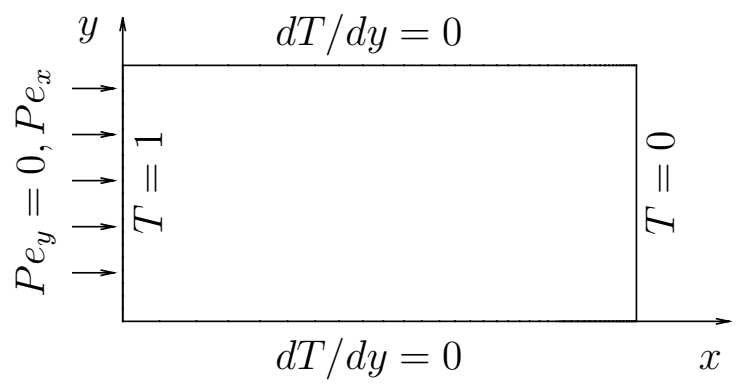

Figure 3: Boundary conditions for entry flow problem. Value of $P e_{x}$ determines the problem.

\subsection{Results}

We compared quadratic nine-node domain element using Lagrangian shape functions, linear for-node domain element using Lagrangian shape functions and 
Table 1: Meshes used in calculation. Meshes are concentrated towards the right side of the domain. Geometric sequence is used where $R$ is the ratio between longest and shortest element.

\begin{tabular}{|c|c|c|}
\hline mesh & nodes $x$ direction & $R$ \\
\hline 1 & 41 & 16 \\
2 & 81 & 16 \\
3 & 161 & 16 \\
4 & 81 & 4 \\
5 & 41 & 4 \\
\hline
\end{tabular}

linear four-node domain element using fourth-order bubble enriched functions using five different meshes. The details of meshes are given in Table 1. Numerical results obtained with ordinary Lagrangian shape function and bubble enriched functions are compared with the analytical solution. In Figure 4 there are results for mesh 2 for Peclet numbers $P e_{x}=20, P e_{x}=50$ and $P e_{x}=200$. The calculated functions accuracy was compared with the analytical values. Due to
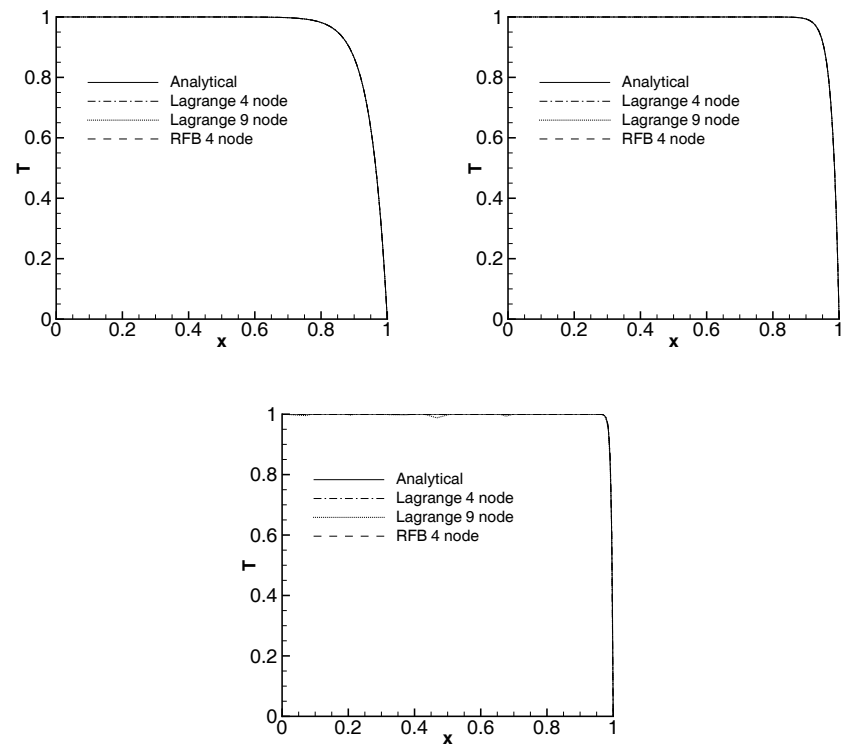

Figure 4: Results for mesh 2, for Peclet number $P e_{x}=20$ (upper left), $P e_{x}=50$ (upper right) and $P e_{x}=200$ (lower). 
high accuracy of the results the different approaches are almost indistinguishable. Thus we introduced the root mean square (RMS) error as:

$$
\text { RMSerror }=\sqrt{\frac{\sum_{i}\left(T_{i}-a_{i}\right)^{2}}{\sum_{i} a_{i}^{2}}}
$$

where $T_{i}$ is the calculated value in node $i$ and $a_{i}$ is the analytical value in node $i$.

Further Figures shows RMS error for different meshes, Peclet numbers and shape functions. Figures 5, 6, 7 show dependence of functions RMS errors for Peclet number $P e_{x}=20, P e_{x}=50, P e_{x}=200$, respectively. The results show decrease of the RMS error with increasing of mesh density. The poorest results are obtained by the Lagrange four node elements. The bubble enriched linear four node elements increase the accuracy for approximately one order of magnitude for the smallest Peclet number. The improvement caused by the bubble enriched elements is less prominent in the case of higher Peclet number values. The quadratic Lagrange nine node elements yield approximately the same accuracy as the bubble enriched linear elements. From results it can be seen, that the residualfree bubble functions add stability to simulation of the entry flow problem.

\section{Conclusions}

Boundary element method with usage of bubble-enriched functions can resolve problems of convective-diffusion and obtain stable and accurate solutions for

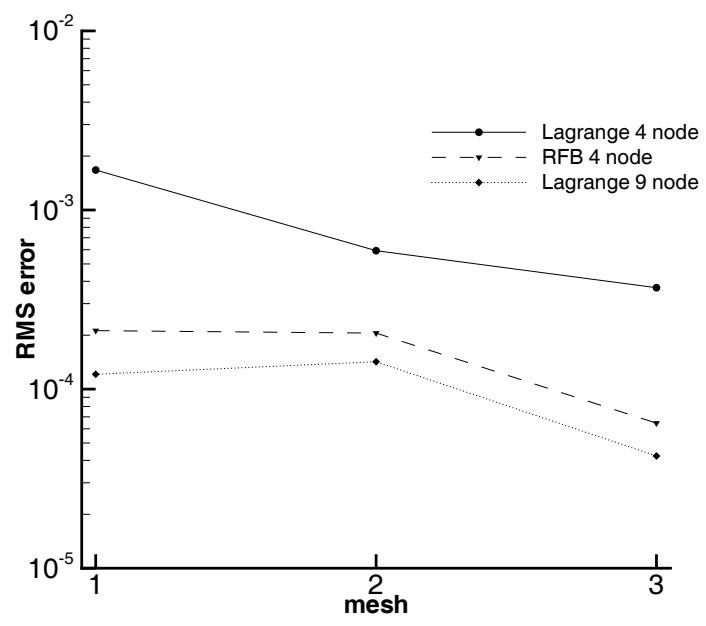

Figure 5: Dependence of functions RMS error to different meshes for Peclet number $P e_{x}=20$. 


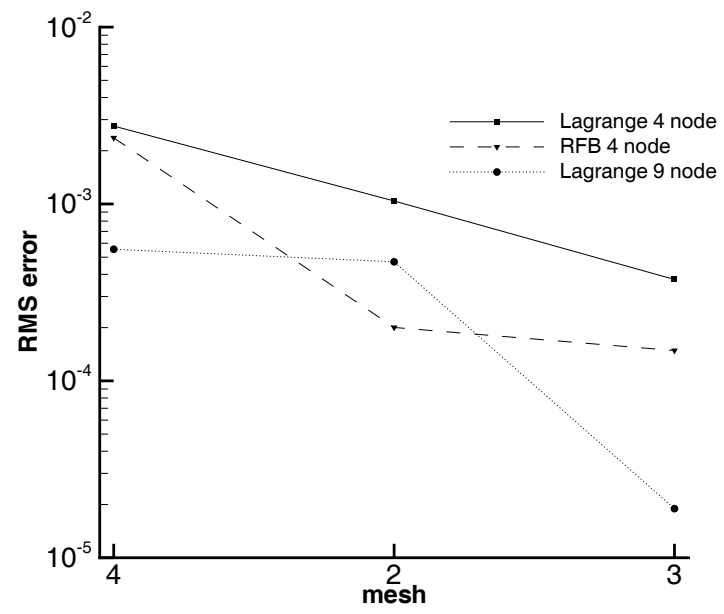

Figure 6: Dependence of functions RMS error to different meshes for Peclet number $P e_{x}=50$.

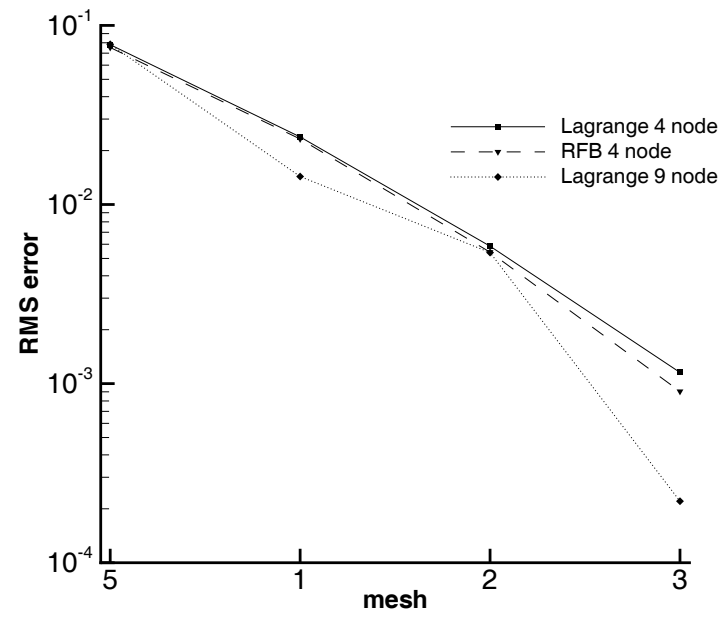

Figure 7: Dependence of functions RMS error to different meshes for Peclet number $P e_{x}=200$.

this type of governing equations, which are being represented in several types of transport phenomena. In the future research, we aim to spread our work on fluid flow simulation. For that purpose we can employ approximate residual-free bubble functions based on polynomial bubble functions. We trust, that combination 
of residual free bubble and convective-diffusion Green's fundamental solution is the most appropriate for solving transport problems where convective respectively hyperbolic part of equation predominate. The mesh compression ratio $R$ also affects the accuracy of the results. It should be chosen appropriately according to the Peclet number and expected function gradients.

\section{References}

[1] N. Samec and L. Škerget. Integral formulation of a diffusive-convective transport equation for reacting flows. Engineering Analysis with Boundary Elements, 28(9):1055-1060, 2004.

[2] V. Nassehi and M. Parvazinia. A multiscale finite element space-time discretization method for transient transport phenomena using bubble functions. Finite Elements in Analysis and Design, 45(5):315-323, 2009.

[3] L.P. Franca, A. Nesliturk, and M. Stynes. On the stability of residual-free bubbles for convection-diffusion problems and their approximation by a two-level finite element method. Computer Methods in Applied Mechanics and Engineering, 166:35-49, 1998. Advances in Stabilized Methods in Computational Mechanics.

[4] L. P. Franca and A. Nesliturk. On a two-level finite element method for the incompressible navier-stokes equations. International Journal for Numerical Methods in Engineering, 52(4):433-453, 2001.

[5] L. Škerget and C.A. Brebbia. Diffusion-convection problems using boundary elements. Advances in Water Resources, 7(2):50-57, 1984.

[6] L. Škerget, M. Hriberšek, and G. Kuhn. Computational fluid dynamics by boundary-domain integral method. Int. J. Numer. Meth. Engng., 46(8):12911311, 1999.

[7] J. Ravnik, L. Škerget, and M. Hriberšek. Two-dimensional velocity-vorticity based LES for the solution of natural convection in a differentially heated enclosure by wavelet transform based BEM and FEM. Engineering Analysis with Boundary Elements, 30(8):671-686, 2006.

[8] L. Škerget, M. Hriberšek, and Z. Žunic. Natural convection flows in complex cavities by BEM. International Journal of Numerical Methods for Heat \& Fluid Flow, 13:720-735, 2003.

[9] Mahmound Parvazinia Vahid Nassehi. Finite Element Modeling of Multiscale Transport Phenomena. Imperial College Press, 2011. 